\title{
Economic load dispatch solutions considering multiple fuels for thermal units and generation cost of wind turbines
}

\author{
Anh Tuan Doan, Dinh Thanh Viet, Minh Quan Duong \\ The University of DaNang-University of Science and Technology, Faculty of Electrical Engineering, \\ DaNang city, Vietnam
}

\begin{tabular}{l}
\hline Article Info \\
\hline Article history: \\
Received Jan 1, 2021 \\
Revised Apr 8, 2021 \\
Accepted Apr 26, 2021 \\
\hline
\end{tabular}

Keywords:

Economic load dispatch Power generation cost Thermal unit Wind farm

\begin{abstract}
In this paper, economic load dispatch (ELD) problem is solved by applying a suggested improved particle swarm optimization (IPSO) for reaching the lowest total power generation cost from wind farms (WFs) and thermal units (TUs). The suggested IPSO is the modified version of Particle swarm optimization (PSO) by changing velocity and position updates. The five best solutions are employed to replace the so-far best position of each particle in velocity update mechanism and the five best solutions are used to replace previous position of each particle in position update. In addition, constriction factor is also used in the suggested IPSO. PSO, constriction factor-based PSO (CFPSO) and bat optimization algorithm (BOA) are also run for comparisons. Two systems are used to run the four methods. The first system is comprised of nine TUs with multiple fuels and one wind farm. The second system is comprised of eight TUs with multiple fuels and two WFs. From the comparisons of results, IPSO is much more powerful than three others and it can find optimal power generation with the lowest total power generation cost.
\end{abstract}

This is an open access article under the $\underline{C C B Y-S A}$ license.

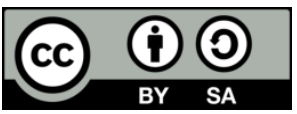

\section{Corresponding Author:}

Minh Quan Duong

Faculty of Electrical Engineering

The University of DaNang-University of Science and Technology

54 Nguyen Luong Bang St., Da Nang city, Vietnam

Email:dmquan@dut.udn.vn

\section{INTRODUCTION}

ELD has been concerned as a huge meaning problem in power system operation. The problem considers power generation cost (PGC) of TUs as the sole target to be minimized effectively. In addition, other requirements from TUs and power system (PS) are also seriously considered such as maximum power generation capability and the balance between electric loads and power sources [1]. Conventional ELD has simplified the main characteristic of TUs as a quadratic function to represent relationship of PGC and power output [2].

The sum of different quadratic functions by using different fuels in thermal power plants was added in the objective function of the problem and it could be solved simply and successfully by conventional methods and modern methods [3]. Conventional methods (CMs) include sub-gradient-based method [4], Newton method [5], [6], and Hopfield neural network [7]. Modern methods are mainly based on metaheuristics such as krill herd algorithm [8], interior search [9], quadratic programming [10], fractal search [11], improved cuckoo search [12], adaptive cuckoo search [13], improved harmony search [14], and hybrid methods such as Nelder-mead and pattern search [15], double elitist breeding quantum-based PSO [16], biogeography-based PSO [17], and Shrink Gaussian distribution-based PSO [18]. As solving ELD for the 
objective, all the mentioned methods have had found approximately the same results and it was hard to identify more robust methods, especially for systems with small number of TUs and without the consideration of power loss in transmission lines (TLs). The challenges become clearer and more significant when another complex factor, which is the operation process of gas or steam valves in TUs, is concerned in the characteristic of TUs. Quadratic function has become a term of PGC function (PGCF) in TUs since another term represented as a sinusoidal function was added. The sum of two terms is the core target of the problem and it has been a challenge for metaheuristics with premature convergence characteristic such as differential evolution (DE) [3] and particle swarm optimization (PSO) [16]. Through results and comparisons analyzed in the previous studies, it can be summarized as shown in:

- CMs are mainly based on the initial points of Lagrange multipliers, partially derivative implementation

- CMs cannot solve complex functions with the presence of sinusoidal PGCF.

- Metaheuristics are widely applied for all functions and they are more effective than CMs. But metaheuristics are easily trapped in local zones with higher or much higher power generation cost than the global optimum.

- Metaheuristics' implementation is time consuming because they have basic parameters (population size and the iteration number) and advanced parameters (mutation factor, scaling factors).

From the analysis above, metaheuristics are preferred rather than CMs but disadvantages of metaheuristics must be eliminated suitably and effectively. Thus, modified versions and hybrid versions of metaheuristics have been developed constantly in recent decades. As optimization tools are developed effectively and fast, ELD problem becomes more complicated since constraints are included in problem formulation such as ramp rate bounds [12] and permitted working zones [13]. In addition, objective function also takes several quadratic functions or the sum of quadratic functions and sinusoidal functions in to account such as the use of multiple fuel options [19], [20] and the consideration of valve-point effect [21], [22].

Nowadays, wind farms (WFs) and photovoltaic systems can produce electricity and they are a part of power sources in power systems with the trend of reducing the use of fossil fuels and cutting the polluted emission into the air. The combination of WFs and TUs is implemented for reaching the lowest PGC [23][25]. Wind speed is supposed to be uncertain as showing a probability function and its generation is influenced by this function. TUs are responsible for generating another part of power after WFs supply electricity to loads. A seven-plant and sixteen-plant combined system with the presence of WFs is solved by PSO and BOA. PSO was less effective than BOA as a conclusion of performance based on the comparison of PGC.

In this paper, WFs and TUs are integrated in power systems to supply electricity to customers. A suggested IPSO and three other methods including PSO, CFPSO and BOA are applied for the problem. The novelty of the paper is as shown in:

- $\quad$ Propose a new PSO method to get higher performance than PSO, CFPSO and BOA;

- $\quad$ Consider multiple fuels for TUs and power generation cost for WFs;

- Consider the penalty cost and reserve cost of WFs.

As solving two systems and comparing results with other methods, the main contributions of the paper are summarized as shown in:

- Successfully develop a real problem of power system operation considering multiple fuels and renewable energy;

- $\quad$ The proposed IPSO method can reach less electric generation cost;

- $\quad$ First combine thermal units with multiple fuels and the uncertainty for wind power;

- $\quad$ Propose an effective method for reaching the best solutions for the considered systems.

\section{MATHEMATICAL FORMULATION OF ELD PROBLEM WITH WFs AND TUS}

The ELD problem with the combined generation of WFs and TUs can be mathematically formulated based on the core objective of reducing PGC and a set of constraints. The combined system is depicted in Figure 1 in which two WFs are represented as two wind farms and two TUs are represented as two thermal power plants.

\subsection{Objective function}

\subsubsection{PGC of TUs}

In this paper, TUs using different fuels are considered for producing and supplying power to loads. So, PGCF is very complicated as shown in (1) and Figure 2. 


$$
P G C_{t}=\left\{\begin{array}{c}
a_{t 1} P T U_{t}^{2}+b_{t 1} P T U_{t}+c_{t 1} \text { for } P T U_{t, 1, \min } \leq P T U_{t} \leq P T U_{t, 1, \max } \\
a_{t 2} P T U_{t}^{2}+b_{t 2} P T U_{t}+c_{t 2} \text { for } P T U_{t, 2, \min } \leq P T U_{t} \leq P T U_{t, 2, \max } \\
\ldots \\
a_{t N 3} P T U_{t}^{2}+b_{t N 3} P T U_{t}+c_{t N 3} \text { for } P T U_{t, N 3, \min } \leq P T U_{t} \leq P T U_{t, N 3, \max }
\end{array}\right.
$$

Where $a_{\mathrm{tN} 3}, b_{\mathrm{tN} 3}, c_{\mathrm{tN} 3}$ are coefficients of PGCF corresponding to the $N 3$ th fuel option of the $t$ th TU; N3 is number of fuel options; $P T U_{t}$ is power generation of the $t$ th $\mathrm{TU}$; and $P T U_{t, N 3, \min }$ are $P T U_{t, N 3, \max }$ are minimum and maximum power generation of the $t$ th $\mathrm{TU}$ for the $N 3$ th fuel option.

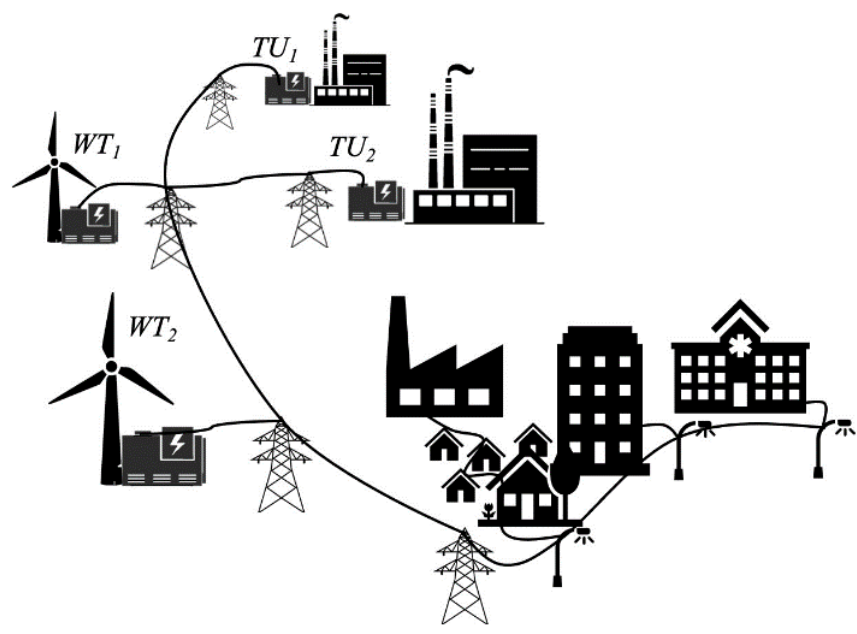

Figure 1. A combined system of wind turbines (WTs) and thermal units (TUs)

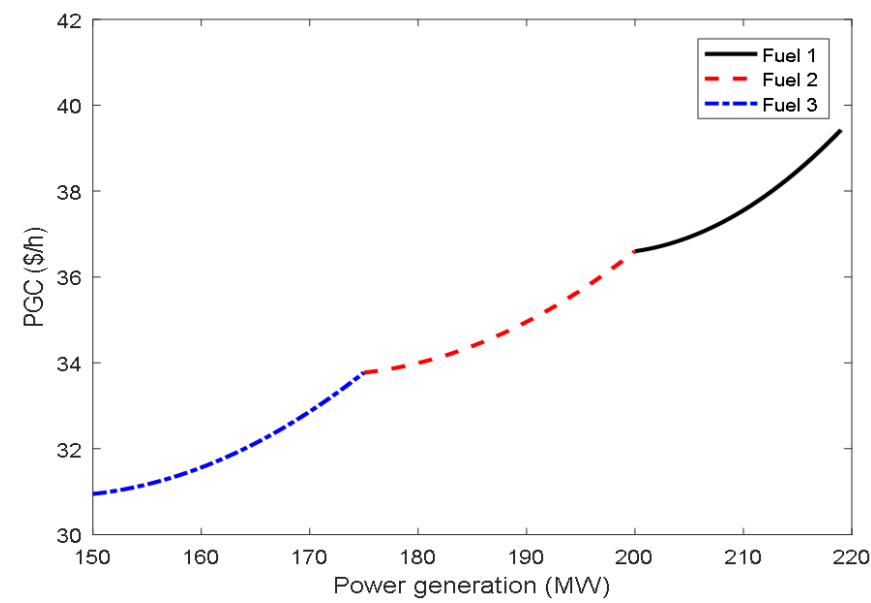

Figure 2. PGFC with three fuel options

As shown in Figure 2, three fuels with three curves are different in terms of power generation and PGC. This is applied to bring the challenge to metaheuristics and it can test the real performance of the suggested IPSO.

\subsubsection{Power generation cost of wind farm}

It is supposed that there are $N 2$ WFs connected in power system and supplying power to loads. Each WF has been paid the cost of $P G C_{w}$, which is calculated by Biswas et al. [26]:

$$
P G C_{w}=D C_{w}+U C_{w}+O C_{w}
$$


Where $D C_{w}$ is direct cost; $U C_{w}$ is penalty cost; and $O C_{w}$ is reserve cost. These costs are obtained by:

$$
\begin{aligned}
& D C_{w}=P r_{d c w} \times P W T_{r w} \\
& U C_{w}=P r_{P w} \times\left(P W F_{r, w}-P W F_{s, w}\right) \\
& O C_{w}=P r_{r w} \times\left(P W F_{s, w}-P W F_{r, w}\right)
\end{aligned}
$$

Where $P r_{d c w}$ is the direct price ( $\$ / \mathrm{MWh}$ ) of the $w t h \mathrm{WF} ; P W F_{r, w}$ is real power output of the $w t h \mathrm{WF} ; P r_{p w}$ is the penalty price $(\$ / \mathrm{MWh})$ of the $w t h \mathrm{WF} ; P W T_{s, w}$ is planned generation of the wth WF; and $P r_{r w}$ is the reserve price $(\$ / \mathrm{MWh})$ of the $w t h \mathrm{WF}$.

\subsubsection{Objective function of the problem}

As shown in two section above, the system with thermal units and wind turbines must pay electric generation cost for fuel cost in thermal units and three other costs in wind power plants. So, the economic issue of the system is to pay the least cost for producing electricity while satisfying all constraints from thermal units and wind power plants. Mathematically, objective function of the problem is to reduce total cost of TUs and WFs and it is expressed as shown in (6):

$$
\text { Reduce } T P G C=\sum_{t=1}^{N 1}\left(P G C_{t}\right)+\sum_{w=1}^{N 2}\left(P G C_{w}\right)
$$

Where $N 1$ and $N 2$ are number of TUs and WFs.

\subsection{Set of constraints}

\subsubsection{Balance of active power}

The balance of active power between power source and load is seriously required. As considering active power loss, this loss and load are added in the same side opposite to power source side. This expression below must be always ensured [27].

$$
\sum_{t=1}^{N 1} P T U_{t}+\sum_{w=1}^{N 2} P W F_{w}-P L o a d-P L o s s=0
$$

On the other hand, power generation from each TU and WF is constrained by [28]:

$$
\begin{aligned}
& P T U_{t, \text { min }} \leq P T U_{t} \leq P T U_{t, \text { max }} \\
& P W F_{w, \text { min }} \leq P W F_{w} \leq P W F_{w, \text { max }}
\end{aligned}
$$

Where $P T U_{t, \min }$ and $P T U_{t, \text { max }}$, and $P W F_{w, \min }$ and $P W F_{w, \max }$ are the minimum and maximum generation of the tth TU and the wth WF.

\section{THE PROPOSED IPSO}

\subsection{Classical PSO}

PSO consists of two core terms, velocity and position as shown in (10) and (11) [29]:

$$
\begin{aligned}
& V_{k}^{\text {new }}=V_{k}+c o_{1} \cdot \varepsilon_{1}\left(M_{\text {best }, k}-M_{k}\right)+c o_{2} \cdot \varepsilon_{2} \cdot\left(M_{\text {best } 1}-M_{k}\right) \\
& M_{k}^{\text {new }}=M_{k}+V_{k}^{\text {new }}
\end{aligned}
$$

Where $V_{k}^{\text {new }}$ and $V_{k}$ are new and old velocities of the $k$ th particle; $M_{k}^{\text {new }}$ and $M_{k}$ are new and old positions of the $k t h$ particle; $\varepsilon_{1}$ and $\varepsilon_{2}$ are random numbers in [0,1]; $\mathrm{co}_{1}$ and $\mathrm{co}_{2}$ are coefficients and set to 2.05; $M_{b e s t, k}$ is the best position of the kth particle; and $M_{b e s t, 1}$ is the best position of the swarm. New velocity update by using (10) is limited due to the fixed change of velocity and constriction factor $K F$ was introduced [30] as shown in (12) and (13):

$$
V_{k}^{\text {new }}=K F\left[V_{k}+c o_{1} \cdot \varepsilon_{1}\left(M_{b e s t, k}-M_{k}\right)+c o_{2} \cdot \varepsilon_{2} \cdot\left(M_{b e s t 1}-M_{k}\right)\right]
$$




$$
K F=\frac{2}{\left|2-\left(\mathrm{co}_{1}+\mathrm{Co}_{2}\right)-\sqrt{\left(\mathrm{co}_{1}+\mathrm{Co}_{2}\right)^{2}-4\left(\mathrm{Co}_{1}+\mathrm{Co}_{2}\right)}\right|}
$$

\subsection{The suggested IPSO method}

In this paper, we suggest using different velocity formulas for each particle by using the five best solutions in the population, called $M_{\text {best }}, M_{\text {best } 2}, M_{\text {best } 3}, M_{\text {best } 4}$ and $M_{\text {best } 5}$. The five solutions are added in a group and then using the random perturbation mechanism to form $M_{r p b e s t}$ from the group. As a result, (12) is modified and written as:

$$
V_{k}^{\text {new }}=K F\left[V_{k}+c o_{1} \cdot \varepsilon_{1}\left(M_{b e s t, k}-M_{k}\right)+c o_{2} \cdot \varepsilon_{2} \cdot\left(M_{r p b e s t}-M_{k}\right)\right]
$$

The new position is also improved by using (15):

$$
M_{k}^{\text {new }}=M_{\text {rpbest }}+V_{k}^{\text {new }}
$$

In this study, the suggested IPSO with the use of (14) and (15) is compared to conventional PSO with the used of (10) and (11) and CFPSO with the use of (11), (12) and (11).

\section{NUMERICAL RESULTS}

In the section, two power systems including TUs and WFs are solved by the suggested IPSO, CFPSO, PSO and BOA. The two systems are modified from a ten-thermal unit system with multiple fuel options for each TU and two wind farms with uncertainty of wind. For running the four methods, population and maximum iteration are set to 20 and 100 for the two systems. Each method is coded in MATLAB programming language and run on a Laptop with the processor of $2.0 \mathrm{Ghz}$ and $4.0 \mathrm{~Gb}$ of RAM. The results from each method are the summary of 50 trials. 50 fuel cost values for 50 trial runs are obtained and then the minimum cost and maximum cost are selected. In addition, the average cost is also calculated by using the 50 cost values. Obtained results and comparisons are presented in the following sections.

\subsection{Results and discussion on the first system}

In this part, the suggested IPSO together with other applied methods are run to solve the first system with the presence of the first nine TUs with multiple fuels and one WF considering related costs such as direct cost, penalty cost and reserve cost. The data of the first nine TUs are taken from [7] with a slight modification that coefficients of PGCF are multiplied by 30. Total load demand is 2,400 MW. The sole WF has the rated power of $120 \mathrm{MW}$ with electric prices are shown in Table 1 [26].

Table 1. Data of the wind farms in the two consider systems

\begin{tabular}{ccccccc}
\hline System & PLoad $(\mathrm{MW})$ & Wind farm $w$ & $\begin{array}{c}P W F_{\text {wmax }} \\
(\mathrm{MW})\end{array}$ & $\begin{array}{c}P r_{d c w} \\
(\$ / \mathrm{MWh})\end{array}$ & $\begin{array}{c}P r_{P w} \\
(\$ / \mathrm{MWh})\end{array}$ & $\begin{array}{c}P r_{r w} \\
(\$ / \mathrm{MWh})\end{array}$ \\
\hline 1 & 2400 & 1 & 120 & 29.7 & 30 & 45 \\
& & 1 & 120 & 36 & 30 & 45 \\
2 & 2400 & 2 & 80 & 35.2 & 30 & 45 \\
\hline
\end{tabular}

Results from 50 implementation times by running four methods are collected. Three main numbers of the results are depicted in Figure 3 and TPGC of 50 runs (sorted from minimum to maximum) are plotted in Figure 4. Three bars of the suggested method are the shortest ones as compared to corresponding bars from others. Furthermore, the deviations among minimum, mean and maximum from the suggested method are very tiny and approximately the same but those from others are extremely higher.

The minimum of CFPSO is better than PSO and BOA but it is still worse than that of the suggested method. The issue indicates that others are searching nearby the global optimum while the suggested method reached this optimum already. Figure 4 confirms the outstanding of the suggested method since all TPGC values of the suggested methods are almost lied on a straight curve but those of others are much higher and increased randomly.

The optimal power generation of all units are shown in Figure 5. Looking through the figure indicates these applied methods found different power generations for the same unit, leading to different PGC values for each unit. But, the results from the suggested method are the best with the lowest TPGC and the highest stability. 


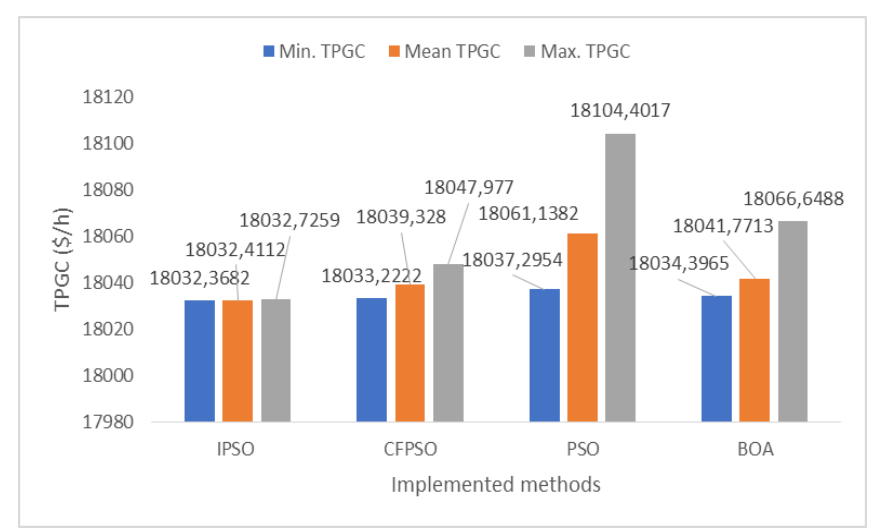

Figure 3. Summary of results for system 1

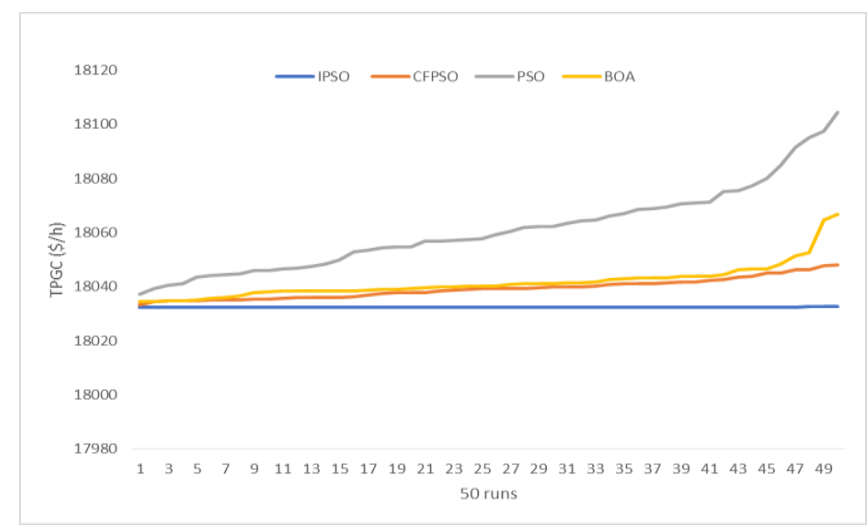

Figure 4. TPGC from 50 runs obtained by executed methods for system 1

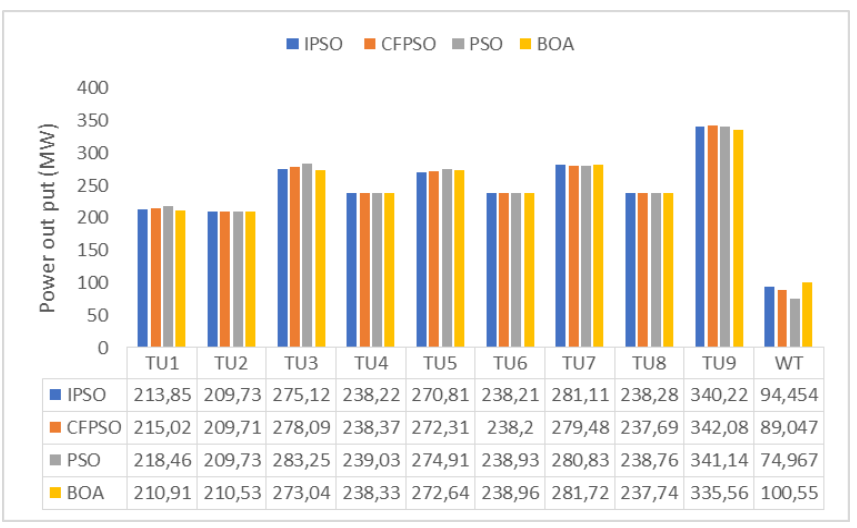

Figure 5. Optimal power of each unit found by four implemented methods for system 1

\subsection{Results and discussion on the second system}

In the part, a more complicated system with the presence of two WFs with the rated power of 120 MW and $80 \mathrm{MW}$ [26]. Prices of two systems are also reported in Table 1. The 9th and last TUs of the system [7] are replaced with the two WFs. Coefficients of PGCF of the eight TUs are taken from [7] and multiplied by 40. The results from 50 implemented times are summarized in Figure 6 and plotted in detail in Figure 7. Figure 6 shows the huge difference from Figure 3 since bars of other ones have very huge deviations and are much higher than ones from the suggested method. The minimum TPGC of the suggested method is the best among minimum from all methods. Furthermore, the mean and maximum TPGC of the suggested method are 
closed to the minimum and much smaller than the minimum of other methods. Namely, the minimum of the suggested method is less than that of CFPSO, PSO and BOA by $\$ 0.9459, \$ 16.3036$ and $\$ 4.2787$, respectively. The mean and maximum of the suggested method are also less than the minimum of others. The mean of IPSO is less than the minimum of CFPSO, PSO and BOA by $\$ 0.9361, \$ 16.2938$ and $\$ 4.2689$. The maximum of IPSO is less than the minimum of CFPSO, PSO and BOA by $\$ 0.7016 \$ 16.0593$ and $\$ 4.0344$, respectively. Clearly, the effectiveness of IPSO is significant. 50 values of TPGC from implemented methods in Figure 7 show the outstanding performance of IPSO over other ones since all TPGC values of IPSO are almost the same and much less than others. The optimal power generations of each TU and WF are presented in Figure 8.

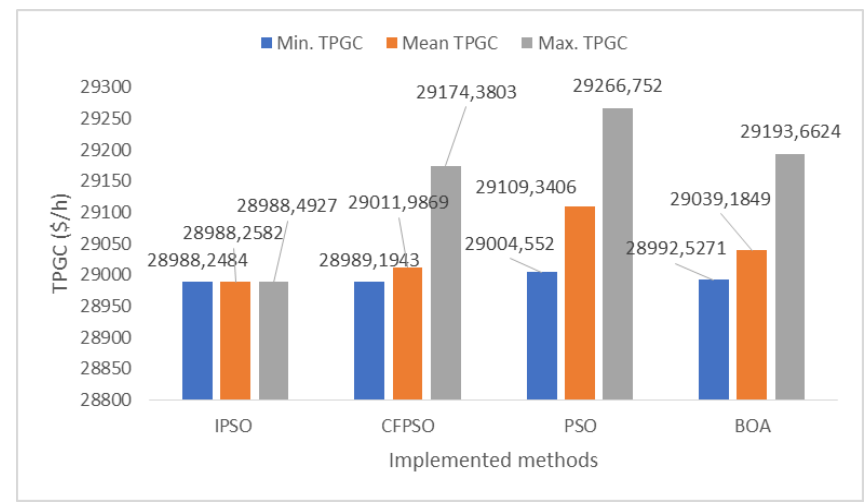

Figure 6. Summary of results for system 2

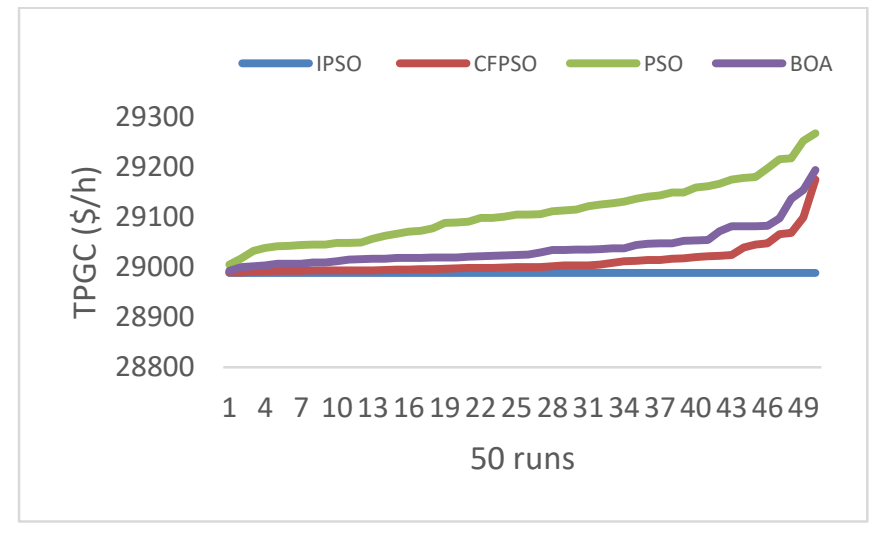

Figure 7. TPGC from 50 runs obtained by executed methods for system 2

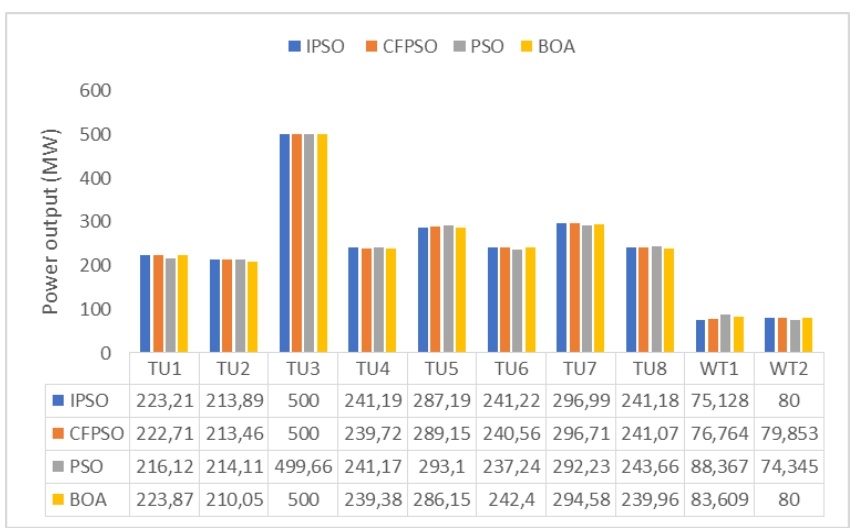

Figure 8. Optimal power of each unit found by four implemented methods for system 2 


\section{CONCLUSION}

In this paper, ELD problem, which has considered multiple fuels of thermal units and three costs of wind farms such as direct cost, penalty cost and reserve cost, has been solved by four methods such as IPSO, CFPSO, PSO and BOA in which IPSO was an improved variant of PSO by using new velocity and position update techniques. The results from a nine-TU and one-WF system and a eight-TU and two-WF system indicated that IPSO was the most powerful one finding the best TPGC for the whole system. Other methods could not find the same best solutions as IPSO and the best TPGC of other methods was also higher than the mean and the maximum TPGC of IPSO. Futhermore, TPGC of IPSO for 50 implemented times was close to the best TPGC with a very tiny difference. As a reuslt, it is stated that the suggested IPSO is very favorable for ELD problem with wind farms and thermal units.

\section{ACKNOWLEDGEMENT}

This research is funded by Vietnam National Foundation for Science and Technology Development (NAFOSTED) under grant number 102.02-2020.07

\section{REFERENCES}

[1] J. Kim, C. S. Kim, and Z. W. Geem, "A memetic approach for improving minimum cost of economic load dispatch problems," Mathematical Problems in Engineering, vol. 2014, no. 1, pp. 1-11, 2014, doi: 10.1155/2014/906028.

[2] P. N. Vinh et al., "Optimal generation for wind-thermal power plant systems with multiple fuel sources," International Journal of Electrical and Computer Engineering (IJECE), vol. 11, no. 2, pp. 1022-1028, 2021, doi: 10.11591/ijece.v11i2.pp1022-1028

[3] X. Shen, D. Zou, X. Zhang, Q. Zhang, and P. Xiao, "A Phase-Based Adaptive Differential Evolution Algorithm for the Economic Load Dispatch Considering Valve-Point Effects and Transmission Losses," Mathematical Problems in Engineering, vol. 2018, no. 9, pp. 1-24, 2018, doi: 10.1155/2018/4585403.

[4] S. K. Gachhayat and S. K. Dash, "Modified sub-gradient based combined objective technique and evolutionary programming approach for economic dispatch involving valve-point loading, enhanced prohibited zones and ramp rate constraints," International Journal of Electrical and Computer Engineering (IJECE), vol 10, no. 5, 2020, Art. no. 5048, doi: 10.11591/ijece.v10i5.pp5048-5057.

[5] K. Navneet, Maninder, and S. Inderjeet, "Economic Dispatch Scheduling using Classical and Newton Raphson Method," International Journal of Engineering and Management Research (IJEMR), vol. 5, no. 3, pp. 711-716, 2015.

[6] C. E. Lin, S. T. Chen and C. Huang, "A direct Newton-Raphson economic dispatch," in IEEE Transactions on Power Systems, vol. 7, no. 3, pp. 1149-1154, Aug. 1992, doi: 10.1109/59.207328.

[7] J. H. Park, Y. S. Kim, I. K. Eom and K. Y. Lee, "Economic load dispatch for piecewise quadratic cost function using Hopfield neural network," in IEEE Transactions on Power Systems, vol. 8, no. 3, pp. 1030-1038, Aug. 1993, doi: $10.1109 / 59.260897$.

[8] A. Bulbul, M. Pradhan, P. K. Roy and T. Pal, "Opposition-based krill herd algorithm applied to economic load dispatch problem," Ain Shams Engineering Journal, vol. 9, no. 3, pp. 423-440, 2018, doi: 10.1016/j.asej.2016.02.003.

[9] I. N. Trivedi, P. Jangir, M. Bhoye, and N. Jangir, "An economic load dispatch and multiple environmental dispatch problem solution with microgrids using interior search algorithm," Neural Computing and Applications, vol. 30, no. 7, pp. 2173-2189, 2018, doi: 10.1007/s00521-016-2795-5.

[10] M. A. Z. Raja, U. Ahmed, A. Zameer, A. Kiani and N. I. Chaudhary, "Bio-inspired heuristics hybrid with sequential quadratic programming and interior-point methods for reliable treatment of economic load dispatch problem," Neural Computing and Applications, vol. 31, no. 1, pp. 447-475, 2019, doi: 10.1007/s00521-017-3019-3.

[11] T. P. Van Hong and T. T. The, "Economic dispatch in microgrid using stochastic fractal search algorithm," GMSARN International Journal, pp. 102-115, Nov. 2017.

[12] T. T. Nguyen, D. N. Vo, and B. H. Dinh, "An effectively adaptive selective cuckoo search algorithm for solving three complicated short-term hydrothermal scheduling problems," Energy, vol. 155, pp. 930-956, 2018, doi: 10.1016/j.energy.2018.05.037.

[13] T. T. Nguyen, D. Vo, N. V. Quynh, and L. V. Dai, "Modified cuckoo search algorithm: A novel method to minimize the fuel cost," Energies, vol. 11, no. 6, pp. 1328, 2018, doi: 10.3390/en11061328.

[14] M. Karthikeyan and T. Sree Ranga Raja, "Dynamic harmony search with polynomial mutation algorithm for valvepoint economic load dispatch," The Scientific World Journal, vol. 2015, doi: 10.1155/2015/147678.

[15] Z. R. Chouhdry, K. M. Hasan, and M. A. Z. Raja, "Design of reduced search space strategy based on integration of Nelder-Mead method and pattern search algorithm with application to economic load dispatch problem," Neural Computing and Applications, vol. 30, no. 12, pp. 3693-3705, 2018, doi: 10.1007/s00521-017-2951-6.

[16] A. Wu and Z.-L. Yang, "An elitist transposon quantum-based particle swarm optimization algorithm for economic dispatch problems," Complexity, vol. 2018, 2018, doi: 10.1155/2018/7276585.

[17] Xu Chen, Bin Xu, and Wenli Du, "An improved particle swarm optimization with biogeography-based learning strategy for economic dispatch problems," Complexity, vol. 2018, no. 2, 2018, doi: 10.1155/2018/7289674. 
[18] L. Ping, J. Sun and Q. Chen, "Solving Power Economic Dispatch Problem with a Novel Quantum-Behaved Particle Swarm Optimization Algorithm," Mathematical Problems in Engineering, vol. 2020, no. 4, pp. 1-11, 2020, doi: 10.1155/2020/9741595.

[19] K. P. Nguyen and G. Fujita, "Self-Learning Cuckoo search algorithm for optimal power flow considering tie-line constraints in large-scale systems," GMSARN International Journal, vol. 12, no. 2, pp. 118-126, 2018.

[20] N. Singh and Y. Kumar, "Multiobjective economic load dispatch problem solved by new PSO," Advances in Electrical Engineering, vol. 2015, no. 1, pp. 1-6, 2015, doi: 10.1155/2015/536040.

[21] M. A. Al-Betar, M. A. Awadallah and M. Krishan, "A non-convex economic load dispatch problem with valve loading effect using a hybrid grey wolf optimizer," Neural Computing and Applications, vol. 32, no. 1, pp. 1-28, 2020, doi: 10.1007/s00521-019-04284-9.

[22] W. Yang, T. Cheng, Y. Guo, Z. Yang and W. Feng, "A Modified Social Spider Optimization for Economic Dispatch with Valve-Point Effects," Complexity, vol. 2020, pp. 1-13, 2020, doi: 10.1155/2020/2865929.

[23] J. Hetzer, D. C. Yu and K. Bhattarai, "An Economic Dispatch Model Incorporating Wind Power," in IEEE Transactions on Energy Conversion, vol. 23, no. 2, pp. 603-611, Jun. 2008, doi: 10.1109/TEC.2007.914171.

[24] J. Tholath Jose, "Economic load dispatch including wind power using Bat Algorithm," 2014 International Conference on Advances in Electrical Engineering (ICAEE), 2014, pp. 1-4, doi: 10.1109/ICAEE.2014.6838546.

[25] K. Chen, L. Han, S. Wang, J. Lu, and L. Shi, "Modified Antipredatory Particle Swarm Optimization for Dynamic Economic Dispatch with Wind Power," Mathematical Problems in Engineering, vol. 2019, pp. 1-17, 2019, doi: $10.1155 / 2019 / 5831362$.

[26] P. P. Biswas, P. N. Suganthan and G. A. J. Amaratunga, "Optimal power flow solutions incorporating stochastic wind and solar power," Energy Conversion and Management, vol. 148, pp. 1194-1207, 2017, doi: 10.1016/j.enconman.2017.06.071.

[27] M. Pradhan, P. K. Roy, and T. Pal, "Oppositional based grey wolf optimization algorithm for economic dispatch problem of power system," Ain Shams Engineering Journal, vol. 9, no. 4, pp. 2015-2025, 2018, doi: 10.1016/j.asej.2016.08.023.

[28] T. Mao Tan, H.-li Yang, B. Duan, Y.-xin Su, and F. He, "Optimizing Production Scheduling of Steel Plate Hot Rolling for Economic Load Dispatch under Time-of-Use Electricity Pricing," Mathematical Problems in Engineering, vol. 2017, pp. 1-13, 2017, doi: 10.1155/2017/1048081.

[29] W. R. Abdul-Adheem, "An enhanced particle swarm optimization algorithm," International Journal of Electrical and Computer Engineering (IJECE), vol. 9, no. 6, pp. 4904-4907, 2009, doi: 10.11591/ijece.v9i6.pp4904-4907.

[30] D. N. Vo, T. P. Nguyen, and K. D. Nguyen, "Multi-Objective Security Constrained Optimal Active and Reactive Power Dispatch Using Hybrid Particle Swarm Optimization and Differential Evolution," GMSARN International Journal, vol. 12, pp. 84-117, 2018. 